\title{
Desensitization Increase As an Additional Mode of Action for NMDA Receptor Open Channel Blockers
}

\author{
M. V. Nikolaev and K. V. Bolshakov \\ Sechenov Institute of Evolutionary Physiology and Biochemistry, Russian Academy of Sciences, \\ ul. M. Toreza, 44, St.Petersburg, 194223 Russia; \\ e-mail: k.bolshakov@biotechnologies.ru
}

DOI: $10.1134 / \mathrm{S} 1990747809030362$

NMDA receptor channel blockers have different mechanisms of interaction with ion channels. "Trapping blockers" remain within ion channel having no influence on gating process. Others, "foot-in-the-door blockers", interfere with gating mechanism, preventing agonist to dissociate while blocker is inside the channel. In the latter case channel blockade compete with desensitization according to the kinetic scheme. Thus blocking mechanism has apparent influence on desensitization, which could mask real influence on desensitization.

In the present work monocationic phenylcyclohexyls IEM-2044 and IEM-2014 and memantine were checked on their direct influence on desensitization. We observed a shift of steady-state desensitization curve to the left in the presence of each blocker, regardless of their action mechanism. More receptors became desensitized in the presence of each blocker. No correlation between the channel blocking mechanism and desensitization increase was observed.

Unlike channel blockade measured at the steadystate level, which was strongly voltage-dependent, influence on desensitization measured at the peak level was voltage independent. Taken together, the results suggest the existence of two different binding sites, which mediate channel blockade and desensitization increase. These data should be taken into account when interpreting clinical data on channel blocker usage. 